\title{
Recovering erasures by using MDS codes over extension alphabets
}

\author{
Sara D. Cardell* Joan-Josep Climent ${ }^{\dagger}$
}

\begin{abstract}
A new family of $\mathbb{F}_{q^{-}}$-linear codes over $\mathbb{F}_{q}^{b}$ can be obtained replacing the elements in the large field $\mathbb{F}_{q^{b}}$ by elements in $\mathbb{F}_{q}[C]$, where $C$ is the companion matrix of a primitive polynomial of degree $b$ and coefficients in $\mathbb{F}_{q}$. In this work, we propose a decoding algorithm for this family of $\mathbb{F}_{q}$-linear codes over the erasure channel, based on solving linear systems over the field $\mathbb{F}_{q}$.
\end{abstract}

Keywords: $\mathbb{F}_{q}$-linear code, companion matrix, primitive polynomial, superregular matrix, erasure channel, linear system

\section{Introduction}

A binary erasure channel (BEC) is a common communications channel model used frequently in information theory because it is one of the simplest channels to analyze and many problems in communication theory can be reduced to a BEC. This channel was introduced by Peter Elias in 1954 [13] as a toy example. In this model, a transmitter sends a bit, and the receiver either receives the bit or it receives an erasure, that is, the bit gets scrambled so the receiver has no idea what the bit was. Unlike the binary symmetric channel, when the receiver gets a bit, it is completely certain that the bit is correct, so the BEC is, in some way, error-free. The only confusion arises when the bit is erased. In this model, a code with minimum distance $d$ can recover up to $d-1$ erasures.

Coding techniques for storage systems have been used widely to protect data against errors or erasure for CDs, DVDs, etc. An increasing number of applications in communication and storage systems uses erasure codes to deal with symbol losses (see, for example, $[10,16]$ ). Assume the data in a storage system is divided into symbols of equal size. Then, an $(n, k)$ block code takes $k$ information symbols and encodes them into $n$ symbols of the same size.

Given a a fixed redundancy, maximum separable codes (MDS codes) offer maximal reliability, since any $k$ symbols are sufficient to recover all the information. Reed-Solomon codes are MDS codes commonly used in storage and communication applications [22, 25]. Another class of MDS codes are MDS array codes [4], for example the EVENODD [1]. In an array code, each symbol consists of a column of elements, and the parities are computed by XORing some information bits. These codes have low computation complexity compared to Reed-Solomon codes, since the encoding and decoding only involve XOR operations [27]. These operations can be implemented by highspeed hardware (for example, $[5,2]$ ). As a consequence, there are many studies that attempt to reduce the high computational complexity of Reed-Solomon codes [7, 18].

Here, we consider block codes over the field $\mathbb{F}_{q^{b}}$, closed under addition and multiplication over $\mathbb{F}_{q}$. These codes are called $\mathbb{F}_{q}$-linear codes over $\mathbb{F}_{q}^{b}[6]$. They can be also seen as array codes over $\mathbb{F}_{q}$ (see [3]). Furthermore, they have practical applications in deep-space communication and computer memory systems $[11,12]$. In the construction proposed in [9], the entries of a superregular matrix in $\mathbb{F}_{q^{b}}$ are replaced by elements in $\mathbb{F}_{q}[C]$, where $C$ is the companion matrix of a primitive polynomial of degree $b$ and entries in $\mathbb{F}_{q}$ (see [8]). Several constructions of MDS block codes based on superregular matrices have been proposed (for example, [24]). The purpose of authors in [9] is to extend these constructions in order to obtain $\mathbb{F}_{q}$-linear codes which are also MDS. Besides, they proposed a decoding scheme over the binary symmetric channel. In this work, we propose a new algorithm to decode this family of codes over the erasure channel. This algorithm recovers up to $n-k$ erasures solving a linear system, whose size depends on the total number of erasures in the information part.

This paper is organized as follows. In Section 2, we remind some preliminaries we need to understand the rest of the paper. In Section 3, we consider the construction of $\mathbb{F}_{q}$-linear codes based on superregular matrices. In

\footnotetext{
*Instituto de Matemática, Estatística e Computação Científica, Universidade Estadual de Campinas (UNICAMP), R. Sérgio Buarque de Holanda, 651 - Cidade Universitária, Campinas - SP, 13083-859, Brazil. sdcardell@gmail.com

${ }^{\dagger}$ Departament de Matemàtiques, Universitat d'Alacant, Ap. Correus 99, E-03080, Alacant, Spain. jcliment@ua.es
} 
Section 4, we introduce the algorithm to decode this family of codes over the erasure channel. Finally, in Section 5 , we give a short conclusion.

\section{Previous Concepts}

Let $\mathbb{F}_{q}$ be the Galois field of $q$ elements and $b$ a positive integer. If $\mathcal{C}$ is a block code of length $n$ over the field $\mathbb{F}_{q^{b}}$, the codewords of $\mathcal{C}$ can be considered as codewords of length $n b$ over $\mathbb{F}_{q}$. Then, a code $\mathcal{C}$ is said to be an $\mathbb{F}_{q}$-linear code of length $n$ over $\mathbb{F}_{q}^{b}$ if it is a linear code of length $n b$ over $\mathbb{F}_{q}$ (see $[6]$ ). The dual code $\mathcal{C}^{\perp}$ is the null space of $\mathcal{C}$ in $\mathbb{F}_{q}^{b}$. The parameter $k=\log _{q^{b}}|\mathcal{C}|$, where $|\mathcal{C}|$ is the number of codewords, is called the normalized dimension of the code over $\mathbb{F}_{q}^{b}$. Recall that the linear space $\mathbb{F}_{q}^{b}$ can be viewed as the field $\mathbb{F}_{q^{b}}$; however, linearity is not assumed over the field $\mathbb{F}_{q^{b}}$. For example, let $\alpha \in \mathbb{F}_{2^{2}}$ be a primitive element such that $\alpha^{2}+\alpha+1=0$, then $\mathbb{F}_{2}^{2}=\{00,01,10,11\} \approx\left\{0,1, \alpha, \alpha^{2}\right\}=\mathbb{F}_{2^{2}}$ and consider the $\mathbb{F}_{2}$-linear code $\mathcal{C}$ of length 2 given by

$$
\mathcal{C}=\{00|00,01| 10,10|01,11| 11\}=\{00,1 \alpha, \alpha 1, \alpha \alpha\}
$$

Since $\alpha(\alpha \alpha)=11 \notin \mathcal{C}$, the code $\mathcal{C}$ is not a linear subspace of $\mathbb{F}_{2^{2}}$.

The code $\mathcal{C}$ can be specified by either its generator matrix $G$ of size $k b \times n b$ or its parity-check matrix $H$ of size $(n-k) b \times n b$, both over $\mathbb{F}_{q}$. From practical considerations, $\mathbb{F}_{q}$-linear codes are required to be systematic, that is, its generator (or parity-check) matrix has to be systematic. Recall that the matrix $G$ (respectively, $H$ ) is said to be systematic if it contains the identity matrix of size $k b \times k b$ (respectively, $(n-k) b \times(n-k) b)$.

To define the minimum distance of an $\mathbb{F}_{q}$-linear code we consider it as a code over the alphabet $\mathbb{F}_{q}^{b}$. Then, the minimum distance $d$ is measured with respect to the symbols in $\mathbb{F}_{q}^{b}$ (see [6]) and thus, the parameters of the code over $\mathbb{F}_{q}^{b}$ are $[n, k, d]$.

A code with parameters $[n, k, d]$ is maximum-distance separable (MDS) over $\mathbb{F}_{q}^{b}$ if the Singleton bound,

$$
d \leq n-k+1
$$

is attained (see $[6,20])$.

In the following example, we show an $\mathbb{F}_{q}$-linear code which is MDS, but the corresponding block code is not MDS.

Example 1: Consider the generator matrix of a linear block code over $\mathbb{F}_{2}$ with length 12 , dimension 4 , and minimum distance equal to 5 ,

$$
G=\left(\begin{array}{ll|ll|ll|ll|ll|ll}
1 & 0 & 0 & 0 & 0 & 1 & 1 & 0 & 0 & 1 & 1 & 0 \\
0 & 1 & 0 & 0 & 1 & 1 & 0 & 1 & 1 & 1 & 0 & 1 \\
\hline 0 & 0 & 1 & 0 & 1 & 1 & 1 & 0 & 1 & 0 & 1 & 1 \\
0 & 0 & 0 & 1 & 1 & 0 & 0 & 1 & 0 & 1 & 1 & 0
\end{array}\right) .
$$

If we consider the code $\mathcal{C}$ over $\mathbb{F}_{2}^{2}$, we have an $\mathbb{F}_{2}$-linear code with length 6 and normalized dimension 2 over $\mathbb{F}_{2}^{2}$. To compute the distance between two codewords, we have to consider the number of different symbols. For example, $d(10|00| 01|10| 01|10,01| 00|11| 01|11| 01)=5$, since these two words only match on the second symbol.

It is not difficult to check that the minimum distance of the $\mathbb{F}_{2}$-linear code $\mathcal{C}$ over $\mathbb{F}_{2}^{2}$ is also 5 . Therefore, $\mathcal{C}$ is an $\operatorname{MDS} \mathbb{F}_{2}$-linear code over $\mathbb{F}_{2}^{2}$.

Note that in this example, the minimum distance is the same in both cases, but they can be different in other cases as we can see in the following example.

Example 2: Consider the $\mathbb{F}_{2}$-linear code $\mathcal{C}$ over $\mathbb{F}_{2}^{2}$ with parameters $[3,1,1]$ and whose generator matrix is given by

$$
G=\left(\begin{array}{ll|ll|ll}
1 & 1 & 0 & 0 & 1 & 1 \\
0 & 0 & 1 & 1 & 0 & 0
\end{array}\right)
$$

If we consider the code as a block code over $\mathbb{F}_{2}$, then the parameters are $[6,2,2]$. So, the $\mathbb{F}_{2}$-linear code and the corresponding block code have not the same minimum distance.

In [8] we can find the following result about the minimum distance of the code over $\mathbb{F}_{q}^{b}$ and over $\mathbb{F}_{q}$.

Theorem 1 (Theorem 1.7 of [8]): Let $\mathcal{C}$ be an $\mathbb{F}_{q}$-linear code with parameters $[n, k]$ over $\mathbb{F}_{q}^{b}$. If $d$ is the minimum distance of the code over $\mathbb{F}_{q}^{b}$ and $D$ is the minimum distance of the code over $\mathbb{F}_{q}$, then $d \leq D$. 
We recall the following result which helps us to check whether an $\mathbb{F}_{q}$-linear code is MDS without computing the minimum distance.

Theorem 2 (Proposition 3.2 of [6]): Let $H=\left(A, I_{(n-k) b}\right)$ be an $(n-k) b \times n b$ systematic parity-check matrix of an $\mathbb{F}_{q}$-linear $[n, k]$ code $\mathcal{C}$ over $\mathbb{F}_{q}^{b}$ and write $A=\left(A_{i, j}\right)$ for $i=1,2, \ldots, k$ and $j=1,2, \ldots, n-k$, where each $A_{i, j}$ is $a b \times b$ block submatrix of $A$. Then $\mathcal{C}$ is $M D S$ if and only if every square submatrix of $A$ consisting of full blocks submatrices $A_{i, j}$ is non-singular.

Next definition reminds the concept of superregular matrix. Different constructions of MDS block codes based on superregular matrices have been proposed in the past (see, for example, [24, 23]).

Definition 1: A matrix $A$ is said to be a superregular matrix over $\mathbb{F}_{q}$ if every square submatrix of $A$ is nonsingular over $\mathbb{F}_{q}$.

For example, the matrix

$$
A=\left(\begin{array}{ll}
1 & 1 \\
2 & 1
\end{array}\right)
$$

is superregular over $\mathbb{F}_{3}$, since every entry is different from 0 and $A$ is non-singular.

As a generalization of this concept, we introduce the concept of block superregular matrix.

Definition 2: A matrix $A$ of size $b m \times b l$ is said to be $b$-block superregular matrix over $\mathbb{F}_{q}$, if every square submatrix of $A$ consisting of full blocks submatrices of size $b \times b$ is non-singular over $\mathbb{F}_{q}$.

Let us see an illustrative example.

Example 3: The matrix

$$
A=\left(\begin{array}{ll|ll}
1 & 0 & 0 & 1 \\
0 & 1 & 1 & 0 \\
\hline 1 & 0 & 1 & 1 \\
0 & 1 & 0 & 1
\end{array}\right)
$$

is a 2-block superregular matrix over $\mathbb{F}_{2}$, since every square submatrix of size $2 \times 2$ is non-singular and $A$ is also non-singular.

Due to Definition 2 and Theorem 2, we can introduce the following theorem.

Theorem 3: Let $H=\left(A, I_{(n-k) b}\right)$ be an $(n-k) b \times n b$ systematic parity-check matrix of an $\mathbb{F}_{q}$-linear code $\mathcal{C}$ with parameters $[n, k]$ over $\mathbb{F}_{q}^{b}$. Then $\mathcal{C}$ is an $M D S \mathbb{F}_{q}$-linear code if and only if $A$ is a b-block superregular matrix over $\mathbb{F}_{q}$.

Now, we can apply the previous theorem in the following example.

Example 4: We consider the $\mathbb{F}_{2}$-linear code $\mathcal{C}$ over $\mathbb{F}_{2}^{2}$ with length 4 and normalized dimension 2, whose paritycheck matrix is

$$
H=\left(A \mid I_{4}\right)=\left(\begin{array}{ll|ll|ll|ll}
1 & 0 & 0 & 1 & 1 & 0 & 0 & 0 \\
0 & 1 & 1 & 0 & 0 & 1 & 0 & 0 \\
\hline 1 & 0 & 1 & 1 & 0 & 0 & 1 & 0 \\
0 & 1 & 0 & 1 & 0 & 0 & 0 & 1
\end{array}\right)
$$

According to Example $3, A$ is a 2-block superregular matrix over $\mathbb{F}_{2}$. Therefore, by Theorem 3 , the $\mathbb{F}_{2}$-linear code $\mathcal{C}$ is MDS.

\section{Construction}

In this section, we recall the construction introduced in [9]. Remember that the companion matrix $C$ of a monic polynomial $p(x)=p_{0}+p_{1} x+\cdots+p_{b-1} x^{b-1}+x^{b} \in \mathbb{F}_{q}[x]$ is the square matrix defined as

$$
C=\left(\begin{array}{ccccc}
0 & 0 & \cdots & 0 & -p_{0} \\
1 & 0 & \cdots & 0 & -p_{1} \\
0 & 1 & \cdots & 0 & -p_{2} \\
\vdots & \vdots & & \vdots & \vdots \\
0 & 0 & \cdots & 0 & -p_{b-2} \\
0 & 0 & \cdots & 1 & -p_{b-1}
\end{array}\right) .
$$


If $p(x)$ is a primitive polynomial, it is well known that $\mathbb{F}_{q^{b}} \approx \mathbb{F}_{q}[C]$, where $\mathbb{F}_{q}[C]=\left\{0, I, C, C^{2}, \ldots, C^{q^{b}-2}\right\}$ (see, for example, [19]).

The isomorphism $\psi: \mathbb{F}_{q^{b}} \rightarrow \mathbb{F}_{q}[C]$, can be defined as $\psi(\alpha)=C$, where $\alpha \in \mathbb{F}_{q^{b}}$ is a primitive element. It can be extended to a ring isomorphism

$$
\Psi: \operatorname{Mat}_{m \times l}\left(\mathbb{F}_{q^{b}}\right) \longrightarrow \operatorname{Mat}_{m \times l}\left(\mathbb{F}_{q}[C]\right)
$$

in the following way: if $A=\left(\alpha_{i j}\right) \in \operatorname{Mat}_{m \times l}\left(\mathbb{F}_{q^{b}}\right)$, then $\Psi(A)=\left(\psi\left(\alpha_{i j}\right)\right) \in \operatorname{Mat}_{m \times l}\left(\mathbb{F}_{q}[C]\right)$. This isomorphism allows us to introduce the following result.

Theorem 4: If $A \in \operatorname{Mat}_{m \times l}\left(\mathbb{F}_{q^{b}}\right)$ is a superrregular matrix, then $H=\left(\Psi(A), I_{b m}\right)$ is the parity check-matrix of an $[m+l, l, m+1] M D S \mathbb{F}_{q}$-linear code $\mathcal{C}$ over $\mathbb{F}_{q}^{b}$.

Remember that if we have an MDS block code, the dual code is MDS as well (see for example [20]). This result can be extended for $\mathbb{F}_{q}$-linear codes. Therefore, the dual code $\mathcal{C}^{\perp}$ of the code constructed in Theorem 4 is an $[m+l, m, l+1] \operatorname{MDS} \mathbb{F}_{q}$-linear code over $\mathbb{F}_{q}^{b}$ as well.

Next example helps us to understand this construction.

Example 5: Consider the primitive polynomial $p(x)=1+x^{2}+x^{3} \in \mathbb{F}_{2}[x]$ whose companion matrix is

$$
C=\left(\begin{array}{lll}
0 & 0 & 1 \\
1 & 0 & 0 \\
0 & 1 & 1
\end{array}\right) \text {. }
$$

Let $\alpha \in \mathbb{F}_{2^{3}}$ be a primitive element such that $1+\alpha^{2}+\alpha^{3}=0$. It is easy to check that $A=\left(\begin{array}{cc}\alpha & \alpha^{4} \\ 1 & \alpha^{2}\end{array}\right)$ is a superregular matrix over $\mathbb{F}_{2^{3}}$. So, according to Theorem 4 , the matrix

$$
H=\left(\begin{array}{cc|c}
C & C^{4} & I_{6} \\
I_{3} & C^{2} &
\end{array}\right)
$$

is the parity-check matrix of an $\mathbb{F}_{2}$-linear code $\mathcal{C}$ over $\mathbb{F}_{2}^{3}$, whose parameters are $[4,2,3]$. Thus, the code is MDS.

If we consider the set of codewords of $\mathcal{C}$ as a linear code over $\mathbb{F}_{2}$, the code is not MDS, since only trivial codes are MDS over $\mathbb{F}_{2}$ [14]. In this case, the parameters of the code are [12,6,3].

The dual code $\mathcal{C}^{\perp}$ is also an MDS $\mathbb{F}_{2}$-linear code over $\mathbb{F}_{2}^{3}$, whose parameters are $[4,2,3]$ as well and whose parity-check matrix is given by

$$
G=H^{\perp}=\left(\begin{array}{l|ll}
I_{6} & \begin{array}{cc}
C^{T} & I_{3} \\
\left(C^{4}\right)^{T} & \left(C^{2}\right)^{T}
\end{array}
\end{array}\right) .
$$

It is worth pointing out that some authors have used the term superregular to define a related but different type of matrices (see for instance $[15,26]$ ). This type of matrices is not suitable to construct MDS $\mathbb{F}_{q}$-linear codes using Theorem 4 , as we will see in the following example.

Example 6: Consider the primitive polynomial $p(x)=x^{3}+x^{2}+1 \in \mathbb{F}_{2}[x]$ whose companion matrix is

$$
C=\left(\begin{array}{lll}
0 & 0 & 1 \\
1 & 0 & 0 \\
0 & 1 & 1
\end{array}\right) \text {. }
$$

Let $\alpha \in \mathbb{F}_{2^{3}}$ be a primitive element such that $\alpha^{3}+\alpha^{2}+1=0$. The matrix $A=\left(\begin{array}{ll}1 & 0 \\ 1 & \alpha\end{array}\right)$ is a superregular matrix over $\mathbb{F}_{2^{3}}$ in the sense of $[15,26]$. Nevertheless, using the isomorphism given by expression (1),

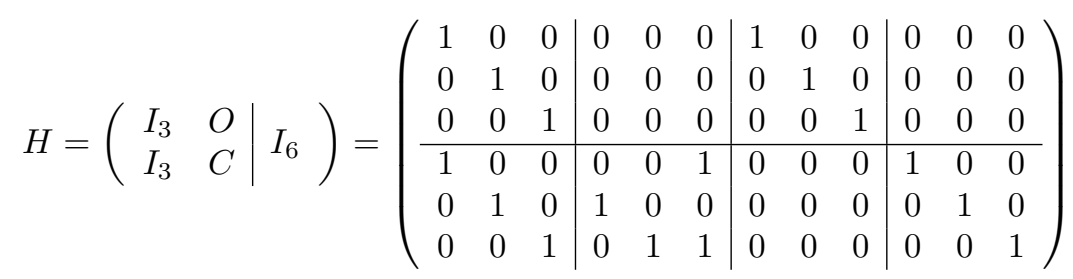

is the parity-check matrix of an $\mathbb{F}_{q}$-linear code $\mathcal{C}$ over $\mathbb{F}_{2}^{3}$ whose parameters are $[4,2,2]$ and therefore, it is not MDS.

The problem now is how to find superregular matrices over a finite field $\mathbb{F}_{q^{b}}$ (see, for example [17, 24, 23]). 


\section{Decoding}

Consider an MDS $\mathbb{F}_{q}$-linear code with length $n$ and normalized dimension $k$ over $\mathbb{F}_{q}^{b}$ and generator matrix $G$ in systematic form. Assume we have $k$ information symbols $\boldsymbol{u}=\left(\begin{array}{llll}\boldsymbol{u}_{1} & \boldsymbol{u}_{2} & \cdots & \boldsymbol{u}_{k}\end{array}\right)$. The corresponding codeword is obtained in the following way

$$
\boldsymbol{v}=\boldsymbol{u} G=\left(\begin{array}{llll|lll}
\boldsymbol{v}_{1} & \boldsymbol{v}_{2} & \cdots & \boldsymbol{v}_{k} & \boldsymbol{v}_{k+1} & \cdots & \boldsymbol{v}_{n}
\end{array}\right) .
$$

Note that $\boldsymbol{v}_{i}=\boldsymbol{u}_{i}$ for $i \in\{1,2, \ldots, k\}$, are the information symbols and $\boldsymbol{v}_{j}$ for $j \in\{k+1, k+2, \ldots, n\}$ are the redundancy symbols.

From now on, we denote by $\langle n\rangle=\{1,2, \ldots, n\}, C_{\mathcal{A}, \mathcal{B}}=\left(C_{a b}\right)$, with $a \in \mathcal{A}$ and $b \in \mathcal{B}$, and $\boldsymbol{v}_{\mathcal{A}}=\left(\boldsymbol{v}_{a}\right)$ with $a \in \mathcal{A}$, where $\mathcal{A}, \mathcal{B} \subseteq\langle n\rangle$.

After transmitting the codeword $\boldsymbol{v}$ through the erasure channel, some of the symbols in $\boldsymbol{v}$ get lost. Let us define the following sets

$$
\begin{aligned}
\mathcal{I} & =\left\{i_{l} \in\langle k\rangle \mid \boldsymbol{v}_{i_{l}} \text { did not get lost during the transmission }\right\}, \\
\mathcal{J} & =\left\{j_{l}-k \mid j_{l} \in\langle n\rangle \backslash\langle k\rangle \text { and } \boldsymbol{v}_{j_{l}} \text { did not get lost during the transmission }\right\}, \\
\mathcal{I}^{c} & =\langle k\rangle \backslash \mathcal{I}=\left\{i_{l}^{c} \in\langle k\rangle \mid v_{i_{l}^{c}} \text { got lost during the transmission }\right\} .
\end{aligned}
$$

In summary, $\mathcal{I}$ and $\mathcal{J}$ are the sets of indices of the correct symbols in the information and redundancy part, respectively, and $\mathcal{I}^{c}$ is the set of indices of the lost symbols in the information part, after transmission. Assume $|\mathcal{I}|=t$ and $|\mathcal{J}|=t^{\prime}$ with $t \leq k$ and $t^{\prime} \leq n-k$. Obviously, the number of erasures in the information part and the redundancy part are $k-t$ and $n-k-t^{\prime}$, respectively and, then, $\left|\mathcal{I}^{c}\right|=k-t$. We do not consider the redundant symbols that are lost during transmission, since they are not necessary to recover the information symbols.

The received symbols $\boldsymbol{v}_{i}, i \in\langle k\rangle$ are the information symbols. However, we only know $t$ of these symbols, $\left(\begin{array}{lllll}\boldsymbol{v}_{i_{1}} & \boldsymbol{v}_{i_{2}} & \cdots & \boldsymbol{v}_{i_{t}}\end{array}\right)$, and we have to recover the other $k-t$ symbols. On the other hand, we know $t^{\prime}$ symbols in the redundancy part, $\left(\begin{array}{lllll}\boldsymbol{v}_{j_{1}} & \boldsymbol{v}_{j_{2}} & \cdots & \boldsymbol{v}_{j_{t^{\prime}}}\end{array}\right)$. Using the known symbols, we can obtain a linear system with $(k-t) b$ unknowns and $t^{\prime} b$ equations,

$$
\boldsymbol{v}_{\langle k\rangle} C_{\langle k\rangle, \mathcal{J}}=\boldsymbol{v}_{\mathcal{J}}
$$

We can find one unique solution as long as $k-t \leq t^{\prime}$, that is, the number of unknowns is less or equal than the number of equations. When $k-t<t^{\prime}$, we have more equations than unknowns, so the system could be incompatible. However, we know $\boldsymbol{u}$ is a solution of the system, so it must be compatible.

We can see the system in (2) as the system

$$
\boldsymbol{v}_{\mathcal{I}^{c}} C_{\mathcal{I}^{c}, \mathcal{J}}+\boldsymbol{v}_{\mathcal{I}} C_{\mathcal{I}, \mathcal{J}}=\boldsymbol{v}_{\mathcal{J}}
$$

where the unknowns are $\boldsymbol{v}_{\mathcal{I}^{c}}$. Let us see an illustrative example.

Example 7: Consider the primitive polynomial $p(x)=1+x+x^{3} \in \mathbb{F}_{2}[x]$, whose companion matrix is given by

$$
C=\left(\begin{array}{lll}
0 & 0 & 1 \\
1 & 0 & 1 \\
0 & 1 & 0
\end{array}\right)
$$

and let $\alpha \in \mathbb{F}_{2^{3}}$ be a root of $p(x)$. Consider the Reed-Solomon code (see [21]) of length 7 and dimension 3 with generator polynomial

$$
g(x)=(x-1)(x-\alpha)\left(x-\alpha^{2}\right)\left(x-\alpha^{3}\right)=\alpha^{6}+\alpha^{5} x+\alpha^{5} x^{2}+\alpha^{2} x^{3}+x^{4} .
$$

A systematic generator matrix of this code is given by

$$
G_{R S}=\left(\begin{array}{ccc|cccc}
1 & 0 & 0 & \alpha & \alpha^{3} & \alpha^{6} & \alpha^{6} \\
0 & 1 & 0 & 1 & \alpha^{4} & \alpha^{2} & \alpha \\
0 & 0 & 1 & \alpha^{2} & \alpha^{5} & \alpha^{5} & \alpha^{6}
\end{array}\right) .
$$

Due to the ring isomorphism considered in expression (1), we can construct an MDS $\mathbb{F}_{2}$-linear code over $\mathbb{F}_{2}^{3}$ with 
parameters $[7,3,5]$ and generator matrix

$$
\begin{aligned}
G & =\left(\begin{array}{cccccc}
P & P^{3} & P^{6} & P^{6} \\
I_{9} & I & P^{4} & P^{2} & P \\
& P^{2} & P^{5} & P^{5} & P^{6}
\end{array}\right) \\
& =\left(\begin{array}{ccccccccc|ccc|ccc|ccc|ccc}
1 & 0 & 0 & 0 & 0 & 0 & 0 & 0 & 0 & 0 & 0 & 1 & 1 & 0 & 1 & 1 & 1 & 0 & 1 & 1 & 0 \\
0 & 1 & 0 & 0 & 0 & 0 & 0 & 0 & 0 & 1 & 0 & 1 & 1 & 1 & 1 & 0 & 0 & 1 & 0 & 0 & 1 \\
0 & 0 & 1 & 0 & 0 & 0 & 0 & 0 & 0 & 0 & 1 & 0 & 0 & 1 & 1 & 1 & 0 & 0 & 1 & 0 & 0 \\
& 0 & 0 & 1 & 0 & 0 & 0 & 0 & 0 & 1 & 0 & 0 & 0 & 1 & 1 & 0 & 1 & 0 & 0 & 0 & 1 \\
0 & 0 & 0 & 0 & 1 & 0 & 0 & 0 & 0 & 0 & 1 & 0 & 1 & 1 & 0 & 0 & 1 & 1 & 1 & 0 & 1 \\
0 & 0 & 0 & 0 & 0 & 1 & 0 & 0 & 0 & 0 & 0 & 1 & 1 & 1 & 1 & 1 & 0 & 1 & 0 & 1 & 0 \\
0 & 0 & 0 & 0 & 0 & 0 & 1 & 0 & 0 & 0 & 1 & 0 & 1 & 1 & 1 & 1 & 1 & 1 & 1 & 1 & 0 \\
0 & 0 & 0 & 0 & 0 & 0 & 0 & 1 & 0 & 0 & 1 & 1 & 1 & 0 & 0 & 1 & 0 & 0 & 0 & 0 & 1 \\
0 & 0 & 0 & 0 & 0 & 0 & 0 & 0 & 1 & 1 & 0 & 1 & 1 & 1 & 0 & 1 & 1 & 0 & 1 & 0 & 0
\end{array}\right) .
\end{aligned}
$$

Consider the information symbols

$$
\boldsymbol{u}=\left(\begin{array}{lll|lll|lll}
1 & 0 & 0 & 0 & 1 & 1 & 1 & 1 & 1
\end{array}\right)
$$

The corresponding codeword is computed in the following way

$$
\boldsymbol{v}=\boldsymbol{u} G=\left(\begin{array}{lll|lll|lll|lll|lll|lll|lll}
1 & 0 & 0 & 0 & 1 & 1 & 1 & 1 & 1 & 1 & 1 & 0 & 0 & 0 & 1 & 1 & 0 & 1 & 0 & 1 & 0
\end{array}\right) .
$$

Assume we receive the following word after transmission

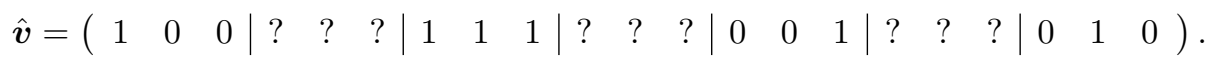

We have erasures in the $2 \mathrm{nd}, 4$ th and 6 th symbols and therefore we have

$$
\mathcal{I}=\{1,3\}, \quad \mathcal{J}=\{5-3,7-3\} \quad \text { and } \quad \mathcal{I}^{c}=\{2\}
$$

The matrix $C_{\langle 3\rangle>, \mathcal{J}}$ is given by

$$
C_{\langle k\rangle, \mathcal{J}}=C_{\langle 3\rangle,\{2,4\}}=\left(\begin{array}{cc}
P^{3} & P^{6} \\
P^{4} & P \\
P^{5} & P^{6}
\end{array}\right)
$$

Therefore, the system (2) is given by

$$
\left(\begin{array}{lll}
\boldsymbol{v}_{1} & \boldsymbol{v}_{2} & \boldsymbol{v}_{3}
\end{array}\right)\left(\begin{array}{cc}
P^{3} & P^{6} \\
P^{4} & P \\
P^{5} & P^{6}
\end{array}\right)=\left(\begin{array}{ll}
\boldsymbol{v}_{5} & \boldsymbol{v}_{7}
\end{array}\right)
$$

The unknown symbol is $\boldsymbol{v}_{2}$ and so

$$
\boldsymbol{v}_{2}\left(\begin{array}{ll}
P^{4} & P
\end{array}\right)=\left(\begin{array}{ll}
\boldsymbol{v}_{5} & \boldsymbol{v}_{7}
\end{array}\right)+\left(\begin{array}{ll}
\boldsymbol{v}_{1} & \boldsymbol{v}_{3}
\end{array}\right)\left(\begin{array}{ll}
P^{3} & P^{6} \\
P^{5} & P^{6}
\end{array}\right)
$$

and therefore, the matrices $C_{\mathcal{I}^{c}, \mathcal{J}}$ and $C_{\mathcal{I}, \mathcal{J}}$ are given by

$$
C_{\mathcal{I}^{c}, \mathcal{J}}=C_{\{2\},\{2,4\}}=\left(\begin{array}{cc}
P^{4} & P
\end{array}\right) \quad \text { and } \quad C_{\mathcal{I}, \mathcal{J}}=C_{\{1,3\},\{2,4\}}=\left(\begin{array}{cc}
P^{3} & P^{6} \\
P^{5} & P^{6}
\end{array}\right),
$$

in this case. Since $\boldsymbol{v}_{\mathbf{2}} \in \mathbb{F}_{2}^{3}$, we have $\boldsymbol{v}_{\mathbf{2}}=\left(\begin{array}{lll}v_{21} & v_{22} & v_{23}\end{array}\right)$. Substituting the actual values in (4) we obtain

$$
\begin{aligned}
\left(\begin{array}{lll}
v_{21} & v_{22} & v_{23}
\end{array}\right)\left(\begin{array}{lll|lll}
0 & 1 & 1 & 0 & 0 & 1 \\
1 & 1 & 0 & 1 & 0 & 1 \\
1 & 1 & 1 & 0 & 1 & 0
\end{array}\right) & =\left(\begin{array}{lll|lll}
0 & 0 & 1 & 0 & 1 & 0
\end{array}\right) \\
& +\left(\begin{array}{llll|ll}
1 & 0 & 0 & 1 & 1 & 1
\end{array}\right)\left(\begin{array}{ccc|ccc}
1 & 0 & 1 & 1 & 1 & 0 \\
1 & 1 & 1 & 0 & 0 & 1 \\
0 & 1 & 1 & 1 & 0 & 0 \\
\hline 1 & 1 & 1 & 1 & 1 & 0 \\
1 & 0 & 0 & 0 & 0 & 1 \\
1 & 1 & 0 & 1 & 0 & 0
\end{array}\right) \\
& =\left(\begin{array}{llll|lll}
0 & 0 & 1 & 1 & 1 & 1
\end{array}\right)
\end{aligned}
$$


which is equivalent to the following system

$$
\left(\begin{array}{lll}
v_{21} & v_{22} & v_{23}
\end{array}\right)\left(\begin{array}{lll}
0 & 1 & 1 \\
1 & 1 & 0 \\
1 & 1 & 1
\end{array}\right)=\left(\begin{array}{lll}
0 & 0 & 1
\end{array}\right)
$$

whose solution is

$$
\boldsymbol{v}_{2}=\left(\begin{array}{lll}
0 & 1 & 1
\end{array}\right)
$$

Recall that $\boldsymbol{u}_{2}=\boldsymbol{v}_{2}$.

Note that the size of the matrix $C_{\mathcal{I}^{c}, \mathcal{J}}$ in system $(3)$ is $(k-t) \times t^{\prime}$. When $k-t<t^{\prime}$, the number of columns in the matrix $C_{\mathcal{I}^{c}, \mathcal{J}}$ is greater than the number of rows. The matrix $C$ is a $b$-block superregular matrix over $\mathbb{F}_{q}$, so every square submatrix composed of blocks of size $b \times b$ is non-singular. Then, if we take the matrix composed by the first $(k-t) b$ columns of $C_{\mathcal{I}^{c}, \mathcal{J}}$, we obtain a non-singular square matrix, $\tilde{C}$, of size $(k-t) b \times(k-t) b$. The remaining columns are a linear combination of these $(k-t) b$ columns. This is the same as removing redundant equations from system (3).

On the other hand, we construct the vector $\tilde{\boldsymbol{v}}$ taking the first $k-t$ symbols of the word $\boldsymbol{v}_{\mathcal{J}}+\boldsymbol{v}_{\mathcal{I}} C_{\mathcal{I}, \mathcal{J}}$ and the solution of system (3) is the same as the solution of the system

$$
\boldsymbol{v}_{\mathcal{I}^{c}} \tilde{C}=\tilde{\boldsymbol{v}}
$$

According to the previous results, we can introduce the following decoding algorithm.

\section{Algorithm 1:}

1. Let $\mathcal{I}$, with $|\mathcal{I}|=k-t$, be the set of the indices of the information symbols that have not been erased during transmission and $\mathcal{J}$, with $|\mathcal{J}|=n-k-t^{\prime}$, the set of the indices of the redundancy symbols, starting by 1 , that have not been erased during transmission. On the other hand, consider the set $\mathcal{I}^{c}$, with $\left|\mathcal{I}^{c}\right|=t$, as the set of the indices of the information symbols that have been erased.

2. If $t+t^{\prime}>k$, it is not possible to recover the lost symbols. Go to step \%. Otherwise, go to next step.

3. Compute the matrices $C_{\mathcal{I}, \mathcal{J}}$ and $C_{\mathcal{I}^{c}, \mathcal{J}}$.

4. Compute the vector $\boldsymbol{v}_{\mathcal{J}}-\boldsymbol{v}_{\mathcal{I}} C_{\mathcal{I}, \mathcal{J}}$.

5. If $t+t^{\prime}=k$, solve the system

$$
\boldsymbol{v}_{\mathcal{I}^{c}} C_{\mathcal{I}^{c}, \mathcal{J}}=\boldsymbol{v}_{\mathcal{J}}-\boldsymbol{v}_{\mathcal{I}} C_{\mathcal{I}, \mathcal{J}}
$$

for $\boldsymbol{v}_{\mathcal{I}^{c}}$ and go to step 7 . Otherwise, go to next step.

6. In this case $t+t^{\prime}<k$. Solve the system $\boldsymbol{v}_{\mathcal{I}^{c}} \tilde{C}=\tilde{\boldsymbol{v}}$, for $\boldsymbol{v}_{\mathcal{I}^{c}}$, where $\tilde{C}$ is the matrix composed by the first $(k-t) b$ columns of $C_{\mathcal{I}^{c}, \mathcal{J}}$, and $\tilde{\boldsymbol{v}}$ is the vector formed by the first $k-t$ symbols of $\boldsymbol{v}_{\mathcal{J}}-\boldsymbol{v}_{\mathcal{I}} \cdot C_{\mathcal{I}, \mathcal{J}}$. Go to step $\%$.

7. End.

The vector $\boldsymbol{v}_{\mathcal{I}^{c}}$, with length $k-t$, is the vector of lost information symbols. Once we compute $\boldsymbol{v}_{\mathcal{I}^{c}}$, we can obtain the complete vector $\boldsymbol{v}$ and thus, the information vector $\boldsymbol{u}$.

\section{Conclusions}

Transmitting $n$ symbols of length $b$ with elements in $\mathbb{F}_{q}$ through an erasure channel is the same as transmitting a codeword of length $n$ of an $\mathbb{F}_{q}$-linear code over $\mathbb{F}_{q}^{b}$. Since proposed codes are MDS, they can recover up to $n-k$ erasures, with $k$ the normalized dimension of the code, over the erasure channel. In this work, we propose an algorithm to recover the erased information symbols by solving a linear system with $(k-t) b$ unknowns, where $t$ in the number of known information symbols.

\section{Acknowledgements}

The work of the first author was partially supported by a grant for postdoctoral students from FAPESP with reference 2015/07246-0. 


\section{References}

[1] Mario Blaum, Jim Brady, Jehoshua Bruck, and Jai Menon. EVENODD: An efficient scheme for tolerating double disk failures in RAID architectures. IEEE Transactions on Computers, 42(2):192-202, 1995.

[2] Mario Blaum, Jehoshua Bruck, and Alexander Vardy. MDS array codes with independent parity symbols. IEEE Transactions on Information Theory, 42(2):529-542, 1996.

[3] Mario Blaum, John L. Fan, and Lihao Xu. Soft decoding of several classes of array codes. In Proceedings of the 2002 IEEE International Symposium on Information Theory (ISIT 2002), page 368, Lausanne, Switzerland, July 2002. IEEE.

[4] Mario Blaum, Patrick G. Farrell, and Henk C. A. van Tilborg. Array codes. In V. S. Pless and W. C. Huffman, editors, Handbook of Coding Theory, pages 1855-1909. Elsevier, North-Holland, 1998.

[5] Mario Blaum and Ron M. Roth. New array codes for multiple phased burst correction. IEEE Transactions on Information Theory, 39(1):66-77, 1993.

[6] Mario Blaum and Ron M. Roth. On lowest density MDS codes. IEEE Transactions on Information Theory, 45(1):46-59, 1999.

[7] Johannes Blömer, Malik Kalfane, Richard Karp, Marek Karpinski, Michael Luby, and David Suckerman. An XOR-based erasure-resilient coding scheme. Technical Report TR-95-048, International Computer Science Institute, Berkeye, CA, 1995.

[8] Sara D. Cardell. Constructions of MDS Codes over Extension Alphabets. PhD thesis, Departamento de Ciencia de la Computación e Inteligencia Artificial, Universidad de Alicante, Alicante, España, August 2012.

[9] Sara D. Cardell, Joan-Josep Climent, and Verónica Requena. A construction of MDS array codes. WIT Transactions on Information and Communication Technologies, 45:47-58, 2013.

[10] Yeow Meng Chee, Charles J. Colbourn, and Alan C. H. Ling. Asymptotically optimal erasure-resilient codes for large disk arrays. Discrete Applied Mathematics, 102:3-36, 2000.

[11] Chin Long Chen. Byte-oriented error-correcting codes for semiconductor memory systems. IEEE Transactions on Computers, 35(7):646-648, 1986.

[12] Chin Long Chen and Brian W. Curran. Switching codes for delta-I noise reduction. IEEE Transactions on Computers, 45(9):1017-1021, 1996.

[13] Peter Elias. Coding for noisy channels. In IRE International Convention Record, part 4, pages 37-46, 1955.

[14] Raymond Hill. A First Course in Coding Theory. Oxford University Press, New York, NY, 1986.

[15] Ryan Hutchinson, Roxana Smarandache, and Jochen Trumpf. On superregular matrices and MDP convolutional codes. Linear Algebra and its Applications, 428:2585-2596, 2008.

[16] Maan A. Kousa and Ali H. Mugaibel. Cell loss recovery using two-dimensional erasure correction for ATM networks. In Proceedings of the Seventh International Conference on Telecommunication Systems, pages 85-89, Nashville, TN, March 1999.

[17] Jérôme Lacan and Jérôme Fimes. A construction of matrices with no singular square submatrices. In Gary L. Mullen, Alain Poli, and Henning Stichtenoth, editors, Finite Fields and Applications, volume 2948 of Lecture Notes in Computer Science, pages 145-147. Springer-Verlag, Berlin, 2003.

[18] Jérôme Lacan and Jérôme Fimes. Systematic MDS erasure codes based on Vandermonde matrices. IEEE Communications Letters, 8(9):570-572, 2004.

[19] Rudolf Lidl and Harald Niederreiter. Introduction to Finite Fields and Their Applications. Cambridge University Press, New York, NY, second edition, 1994.

[20] Florence Jessie MacWilliams and Neil James Alexander Sloane. The Theory of Error-Correcting Codes. NorthHolland, Amsterdam, 6 edition, 1988. 
[21] Irving S. Reed and Gustave Solomon. Polynomial codes over certain finite fields. Journal of the Society for Industrial and Applied Mathematics, 8(2):300-304, 1960.

[22] Steven Roman. Coding and Information Theory. Springer, New York, NY, 1992.

[23] Ron M. Roth and Abraham Lempel. On MDS codes via Cauchy matrices. IEEE Transactions on Information Theory, 35(6):1314-1319, 1989.

[24] Ron M. Roth and Gadiel Seroussi. On generator matrices of MDS codes. IEEE Transactions on Information Theory, 31(6):826-830, 1985.

[25] Peter Sweeney. Error Correcting Coding. John Wiley \& Sons, West Sussex, England, 2002.

[26] Virtudes Tomás, Joachim Rosenthal, and Roxana Smarandache. Decoding of convolutional codes over the erasure channel. IEEE Transactions on Information Theory, 58(1):90-108, 2012.

[27] Zhiying Wang, Alexandros G. Dimakis, and Jehoshua Bruck. Rebuilding for array codes in distributed storage systems. In Proceedings of the IEEE Globecom 2010 Workshop on Application of Communication Theory to Emerging Memory Technologies, pages 1909-1909, Miami, FL, December 2010. IEEE. 